\title{
DESARROLLO DE MODELOS DE ANÁLISIS NUMÉRICO MEDIANTE LA UTILIZACIÓN DE ARCHIVOS DE INTERCAMBIO
}

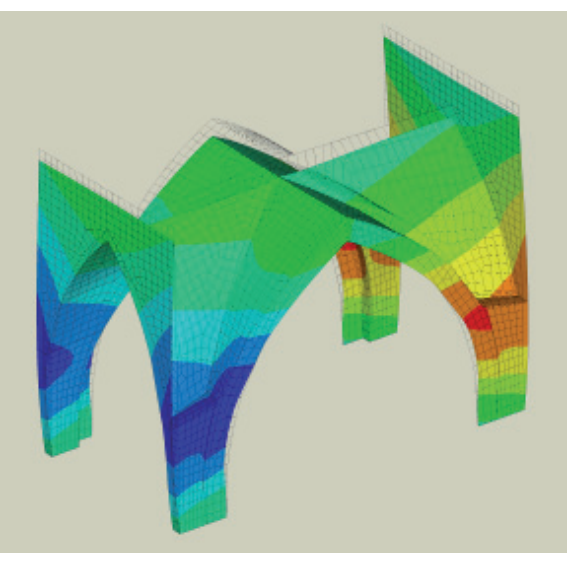

\author{
Emilio Martín Gutiérrez y Juan B. Pérez Valcárcel
}

Models of Numerical Analysis Using Exchange Files

Boletín Académico. Revista de investigación y arquitectura contemporánea
Escuela Técnica Superior de Arquitectura. Universidade da Coruña
elSSN 2173-6723
www.boletinacademico.com
Número 2 (2012)
Páginas 46-55
Fecha de recepción 17.10.2011
Fecha de aceptación 24.01.2012

https://doi.org/10.17979/bac.2012.2.0.976

\section{Resumen}

El presente artículo describe los avances efectuados en el ámbito de la generación de modelos de análisis numérico, combinando técnicas de diseño asistido por ordenador y de programación para lograr un intercambio de información entre aplicaciones de diferente propósito. Esto permite mejorar el proceso, usando en cada fase las herramientas que específicamente pueden optimizarla. El planteamiento ha resultado especialmente útil en estudios de estabilidad realizados sobre edificaciones con valor histórico o con singularidades geométricas de todo tipo. En estos casos, la gran complejidad del modelo dificulta extraordinariamente su introducción, si sólo se utilizan las herramientas de preproceso convencionales.

\begin{abstract}
This work describes the advancements made in the development of models of numerical analysis, combining Computer-Aided Design (CAD) and programming to exchange information between different applications. This allows improvements in the process, given specific tools can be used to optimize each phase. This approach has been especially useful in studies of stability performed on historical buildings. In these cases it is extremely difficult to develop a model given its complexity if only conventional preprocessing tools are to be used.
\end{abstract}

\section{Palabras clave}

Archivos de intercambio, modelos mecánicos, análisis numérico.

\section{Keywords}

Exchange files, mechanical models, numerical analysis. 


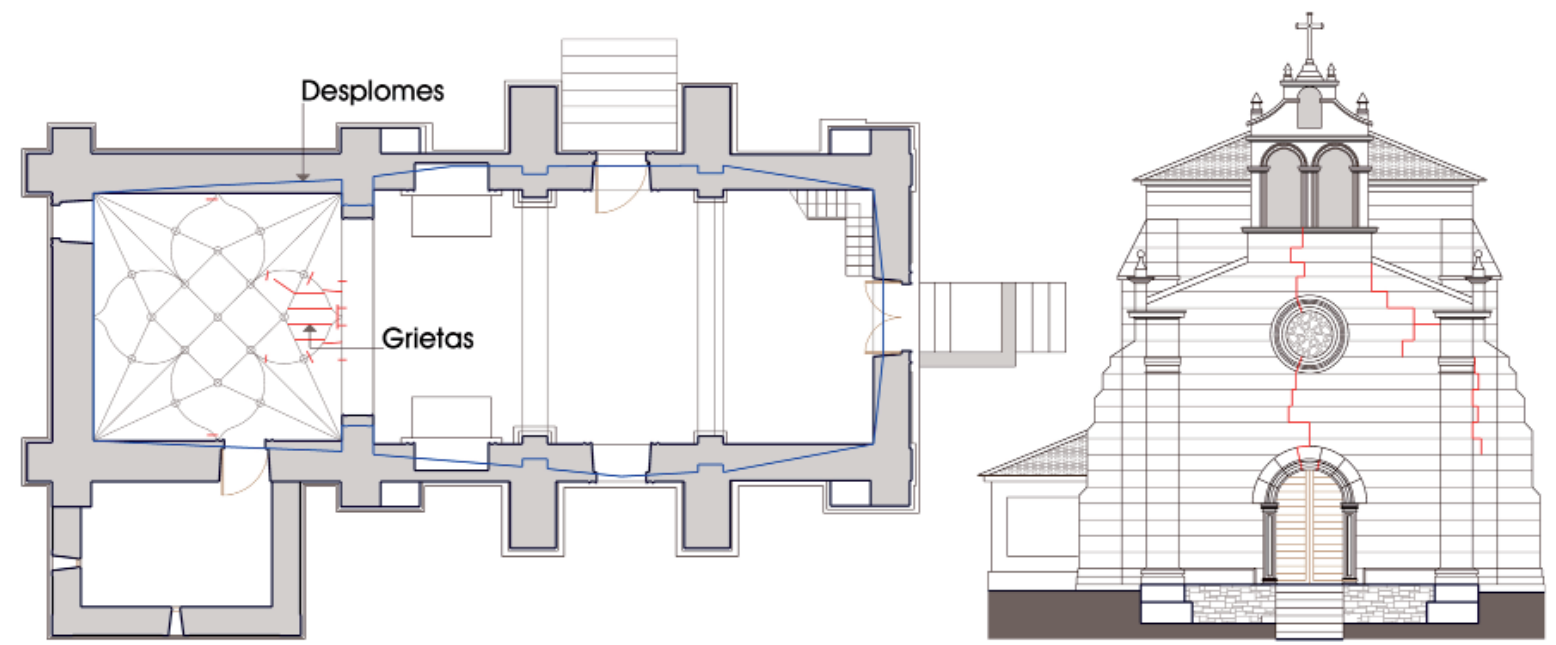

1 Iglesia de santa María, Guimarei (Lugo, S. XIV-XVII). Desplomes y fisuras en paramentos interiores y fachadas.

\section{INTRODUCCIÓN}

En el momento actual, el arquitecto debe utilizar en el ejercicio de su profesión numerosos programas informáticos de diferente propósito (diseño gráfico, bases de datos, hojas de cálculo, análisis estructural, mediciones y presupuestos, etc.). Generalmente, es más útil emplear herramientas específicas diseñadas para cada tarea que intentar ceñirse a una aplicación de enfoque global. Por ello, la capacidad de intercambiar información entre programas resulta cada vez más necesaria.

Dentro de la investigación desarrollada por nuestro equipo, ha resultado especialmente útil el intercambio entre sistemas de $C A D$ y programas de cálculo. En campos muy específicos, los últimos implican tareas de cierta complejidad, especialmente en lo que respecta a la definición geométrica y mecánica de los modelos. Por el contrario, la confección de los mismos bajo un entorno gráfico, y su posterior trasvase a una aplicación de análisis, no sólo agiliza las correspondientes operaciones, sino que facilita igualmente un mayor control sobre las características del modelo y su adecuación al sistema que se pretende estudiar.

De entre los diferentes sistemas de intercambio, el denominado DXF (registrado por Autodesk Inc) ${ }^{1}$ continúa siendo una referencia desde sus inicios. La menor compacidad de los archivos asociados queda plenamente compensada por el hecho de poder expresarse íntegramente en código $A S C I I$, de forma que es posible proceder a su manipulación con ayuda de un simple editor de texto convencional.

\section{ANÁLISIS NUMÉRICO DE CONSTRUCCIONES SINGULARES. CONSIDERACIONES INICIALES}

Desde el año 1995, el Departamento de Tecnología de la Construcción de la Universidad de A Coruña viene realizando estudios de estabilidad y análisis de comportamiento estructural sobre edificios con valor histórico. Se trata de trabajos multidisciplinares en los que se analiza históricamente el edificio, se aborda un levantamiento gráfico, se estudian sus posibles procesos patológicos (Fig. 01), y se confeccionan modelos numéricos con objeto de analizar los problemas detectados y, en su caso, valorar soluciones alternativas.

En general, los datos del edificio se representan en un entorno de diseño asistido por ordenador, mientras que el análisis estructural se efectúa por el método de los elementos finitos (MEF), mediante operaciones que a su vez se estructuran en tres grandes bloques:

- Preproceso. Donde se definen los tipos de elementos y materiales constitutivos, se configura la geometría del modelo, y se efectúa el mallado del mismo con arreglo a determinadas especificaciones.

- Cálculo. En esta segunda etapa se asignan los correspondientes vínculos, se aplican las acciones, y se aborda propiamente el cálculo del sistema.

- Postproceso. Facilita la gestión gráfica y documental de los resultados obtenidos previamente. 


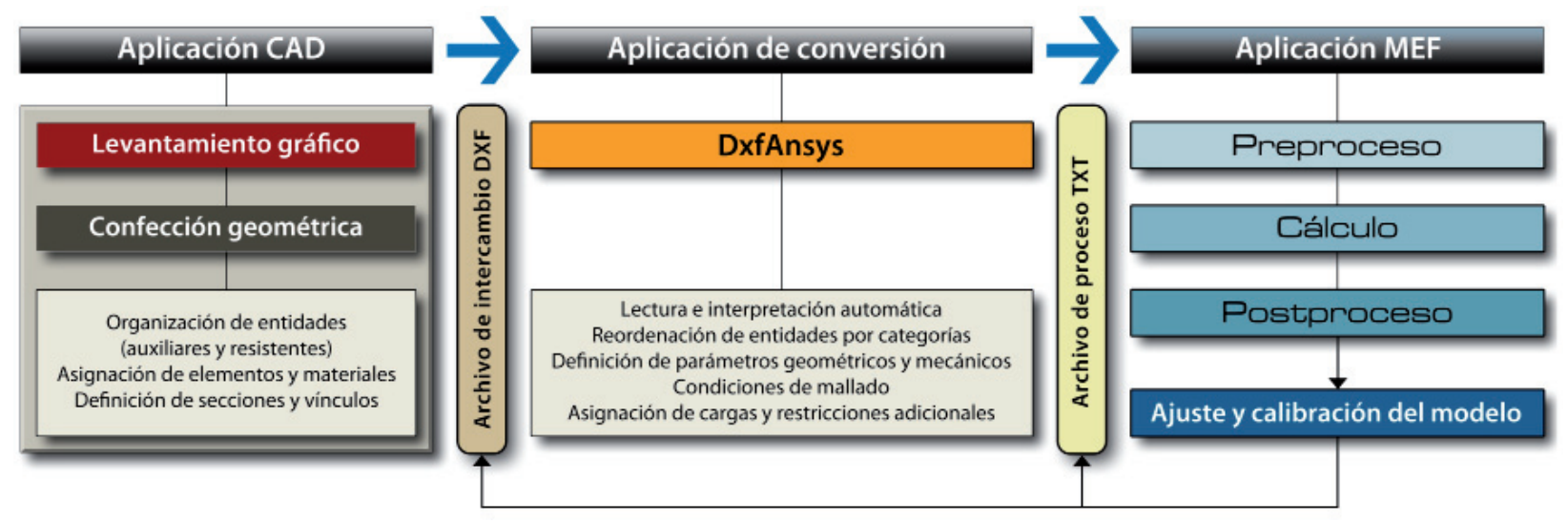

2 Mapa conceptual relativo a la técnica propuesta para la confección y posterior análisis de modelos.

Durante la fase inicial se confecciona la geometría del modelo mediante la definición —a menudo secuencial- de puntos, líneas, áreas y volúmenes, recurriendo a un catálogo de comandos y herramientas, en general, bastante alejado de los recursos habituales en entornos de diseño asistido. El proceso, en conjunto, resulta complejo, lento y de difícil control, por lo que sólo reviste utilidad para geometrías simples. En el campo de las edificaciones medievales, con geometrías complejas e irregulares, esta técnica no resulta operativa en modo alguno.

A tales efectos, muchas aplicaciones MEF incorporan sistemas de intercambio de la información gráfica previamente generada en un entorno $C A D$. En nuestro caso, el programa utilizado, Ansys Multiphysics ${ }^{2}$, incorpora una alternativa basada en el formato IGES. No obstante, este sistema resulta complejo en lo que respecta a su manipulación y, por encima de otras consideraciones, no permite transmitir más que los datos netamente geométricos. De esta forma, aspectos tan relevantes como la definición de los parámetros mecánicos, los tipos de elementos a considerar en la discretización, las condiciones de mallado, o las coacciones y cargas que solicitan al modelo, no se podrían gestionar desde el entorno gráfico. Para incorporar todos estos objetivos, nuestro equipo ha desarrollado una aplicación intermedia, DxfAnsys ${ }^{3}$, que permite transferir la totalidad de datos que definen el modelo, en sus aspectos geométrico y mecánico, desde la aplicación $C A D$ al paquete informático MEF por medio de un archivo de intercambio $D X F$.
Con dicha herramienta, el proceso se puede estructurar, en líneas generales, del siguiente modo (Fig. 02): tomando como base el levantamiento gráfico previo de plantas, alzados y secciones (necesario en todo programa de intervención), se construye la geometría del modelo empleando un determinado catálogo de entidades (aspecto perfilado en los siguientes epígrafes), siempre recurriendo a herramientas habituales que no requieren un aprendizaje específico. En el mismo entorno gráfico es posible codificar materiales, elementos, vínculos y acciones, utilizando nuevamente recursos de tipo gráfico. Este primer trazado se exporta en formato $D X F$, que a su vez se gestiona con ayuda de la aplicación DxfAnsys. En esta etapa se reordenan automáticamente las entidades del modelo (con objeto de facilitar su posterior localización), se reinterpreta y complementa la información asociada, y se definen las condiciones de mallado. Este procedimiento conduce a un nuevo archivo en formato $A S C I I$, que es posible procesar de forma autónoma y secuencial mediante Ansys.

En los siguentes apartados se describe en términos genéricos la metodología propuesta. Hasta el momento, la técnica se ha utilizado satisfactoriamente en diversos estudios de carácter histórico ${ }^{4}$. Por otro lado, su aplicación ha resultado sumamente útil en el análisis de diferentes construcciones contemporáneas, cuyas singularidades de proyecto exigían procedimientos $y$ herramientas menos habituales en el ámbito de la edificación ${ }^{5}$ (Fig. 03). 


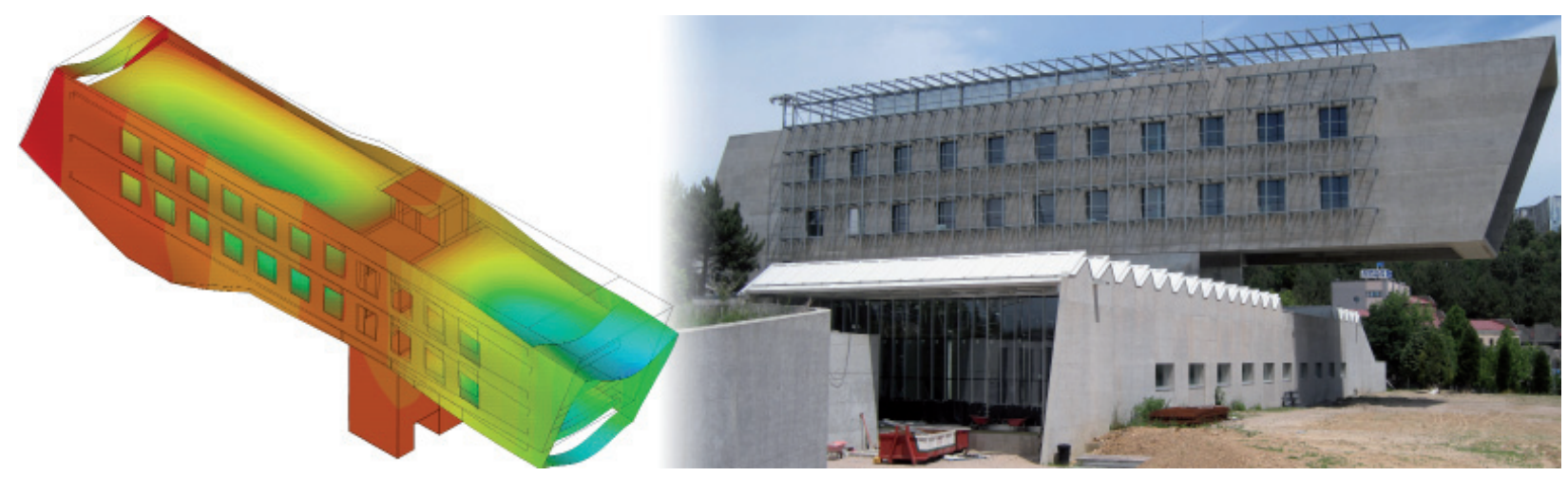

3 Alfonso Penela Fernández, Módulo Tecnológico Industrial de la Universidad de Vigo, Vigo (Pontevedra, 2008). Modelo de análisis e imagen obtenida durante su construcción.

\section{ESTRUCTURA BÁSICA DEL FORMATO DXF (ASCII)}

Los archivos de intercambio con formato DXF se componen de secuencias de dos líneas consecutivas. La primera presenta una cifra denominada código de grupo, que a su vez sirve para interpretar el contenido de la segunda. Cada una de estas parejas de datos se integra en secciones, de modo que la estructura resultante facilita la identificación de los elementos pertinentes en cada operación. La secuencia general de secciones responde a la siguiente ordenación:

- Header: presenta el número de la versión utilizada $\mathrm{y}$ una serie de variables del sistema.

- Clases: vinculada a las clases definidas por la aplicación, y cuyas apariciones se registran en las secciones blocks, entities y objects posteriores.

- Tables: contiene las tablas de símbolos asociadas a estilos de cota, configuración de capas, tipos de línea, estilos de texto, sistemas de coordenadas personales, y aspectos de visualización, entre otras cuestiones.

- Blocks: define las características ligadas a cada uno de los bloques del dibujo, cuyas posibles inserciones se integran en la sección subsiguiente.

- Entities: en este sector se alojan todos los elementos gráficos del dibujo, incluyendo aquellas entidades que servirán para configurar el modelo de cálculo. Por ello, la aplicación intermedia que interpreta el archivo de intercambio dirige su atención directamente al contenido de este apartado.

- Objects: relativa a los objetos no gráficos de la base de datos.

- Thumbnailimage: sección opcional relativa a la vista preliminar del dibujo.

Dentro de la sección Entities se utilizan determinados códigos de grupo, que a su vez definen diversas propiedades de los objetos (capa, tipo de línea, color, grosor, escala, etc.). Estos parámetros son utilizados por la aplicación de conversión para gestionar aspectos tan relevantes como la asignación de elementos y materiales, los criterios de mallado o la aplicación de cargas.

En lo que sigue, se describe sucintamente el tratamiento de las entidades que configuran el modelo, $y$ las particularidades que cabe contemplar en su definición para asegurar su correcta interpretación en la fase final de análisis.

\section{LECTURA E INTERPRETACIÓN DE ENTIDADES}

El primer objetivo consiste en abordar la necesaria definición geométrica utilizando los recursos de un entorno de diseño asistido convencional, y empleando como base de trabajo el levantamiento previo de la edificación (Fig. 04). En este sentido, se hace preciso asociar las posibles entidades gráficas disponibles en 


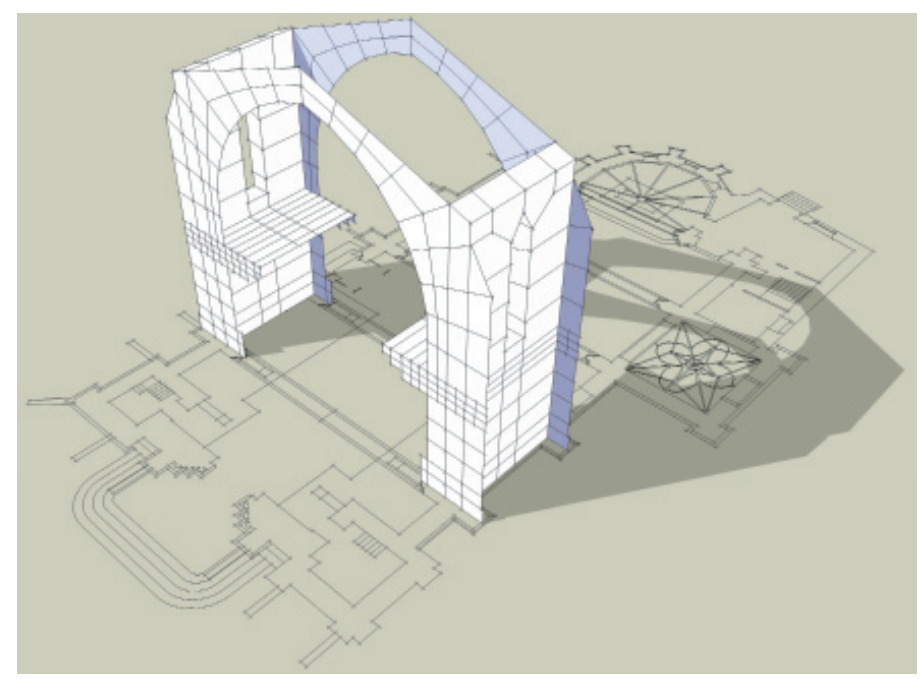

4 Iglesia de san Martín, Noia (A Coruña, s. XIII). Construcción del modelo global bajo un entorno de diseño asistido.

el sistema $C A D$ con los objetos que pueden configurar un modelo de cálculo reconocible por la aplicación MEF. Éste último, desde un punto de vista netamente geométrico, está constituido por puntos, líneas (rectas y arcos de circunferencia), áreas y volúmenes. Con estas categorías es posible definir conjuntos de cierta complejidad (Fig. 05-06), al menos a fin de obtener una aproximación cualitativa a su comportamiento resistente.

No obstante, en esta fase se requiere distinguir aquellos elementos con responsabilidad mecánica de otros auxiliares de trazado. Con este fin, se adopta el siguiente criterio: los elementos que definen la estructura (nervios, columnas, plementería, contrafuertes, muros, etc.) se disponen en capas con designación numérica; de forma que todos los incluidos en una misma capa estarán constituidos por un mismo material y modelizados con un mismo tipo de elemento finito. Por el contrario, las capas con designación alfanumérica se considerarán auxiliares de cara a la gestión geométrica del modelo, pero no se procesarán como fracciones resistentes del mismo.

Los puntos clave que integran la geometría básica no requieren una definición expresa, toda vez que se reconoce su posición a partir de los restantes elementos: extremos de línea, límites y centros de arcos, y vértices de polilíneas y $3 \mathrm{D}$-caras. Cuando en el proceso de conversión e intercambio se detecta uno de estos puntos, se hace preciso verificar si existe algún otro previamente definido en la misma posición. En tal caso, basta referenciar la nueva entidad a la numeración identificativa de los puntos previamente localizados, lo que asegura, en el modelo matemático, una adecuada conexión entre las diferentes partes que lo componen.

Cada una de las entidades constitutivas debe ser ubicada en el archivo de intercambio, dentro de la sección Entities, detectando un código de grupo 0 seguido por la información correspondiente a cada tipo (line, arc, polyline). Durante el proceso de conversión, la aplicación informática intermedia reconoce los parámetros asociados a cada objeto, y los almacena ordenadamente en matrices de tipo dinámico. En esta etapa se introducen asimismo rutinas automatizadas que previenen la comisión de determinados errores o garantizan una adecuada configuración geométrica y matemática: localización de posibles líneas de longitud nula, gestión de aristas coincidentes entre elementos contiguos, etc.

En esta fase, el aspecto de mayor complejidad es precisamente el tratamiento de los arcos. Para reducir espacio en la base de datos gráfica, las aplicaciones $C A D$ resuelven su almacenamiento con relación al denominado sistema de coordenadas de objeto, y que responde a un algoritmo interno por el cual se definen unos ejes arbitrarios asociados a cada entidad. En consecuencia, es preciso comprender dicho algoritmo y efectuar una conversión al sistema de coordenadas global, de forma que la definición geométrica del arco resulte compatible con el preprocesador de la aplicación MEF.

En lo que respecta a las áreas, se han previsto dos modalidades de introducción, en función del tipo de tra- 


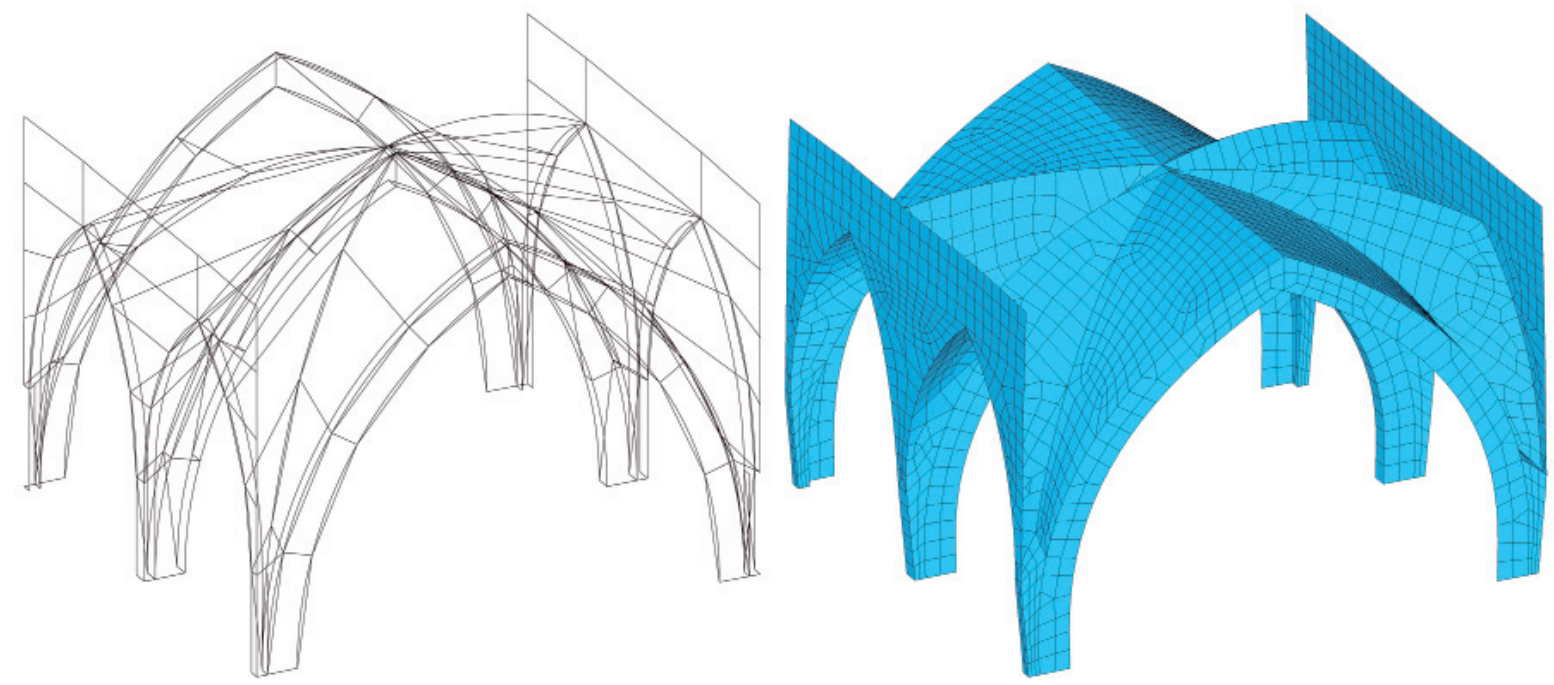

5 Catedral de Saint-Étienne (Bourges, s. XII-XIII). Modelo de bóveda sexpartita.
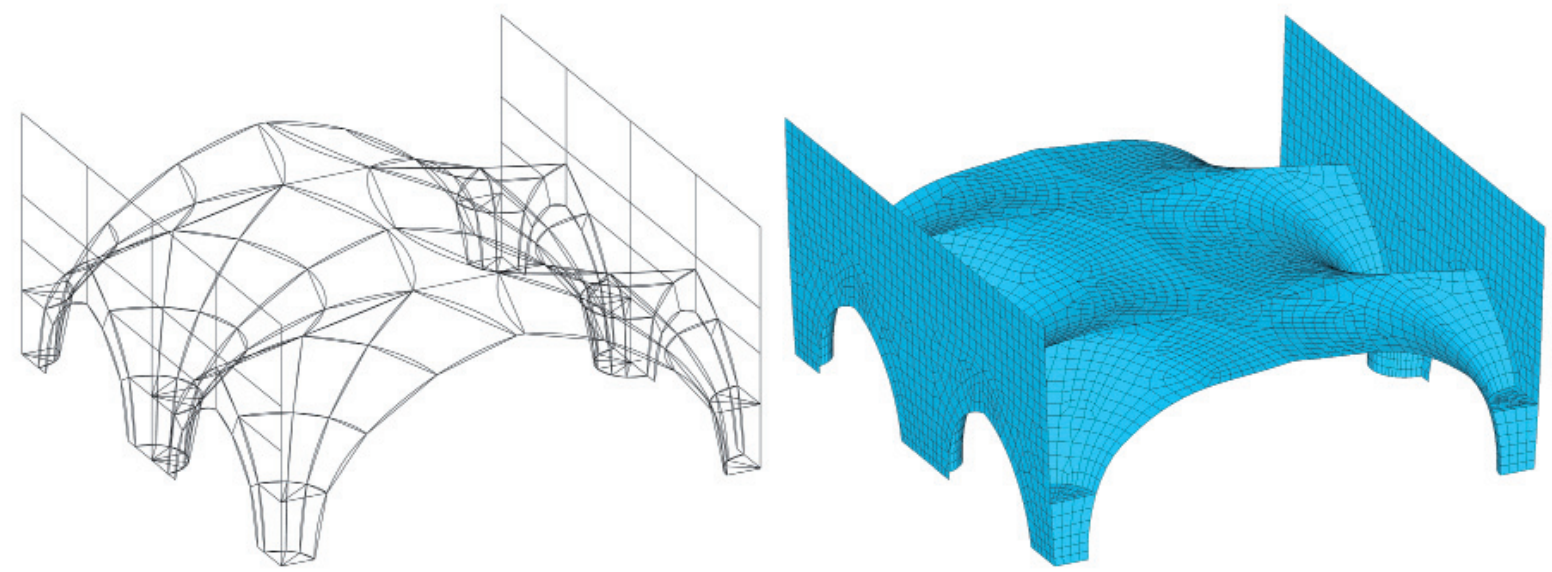

6 Capilla del King's College (Cambridge, 1446-1531). Modelo de bóveda en abanico.
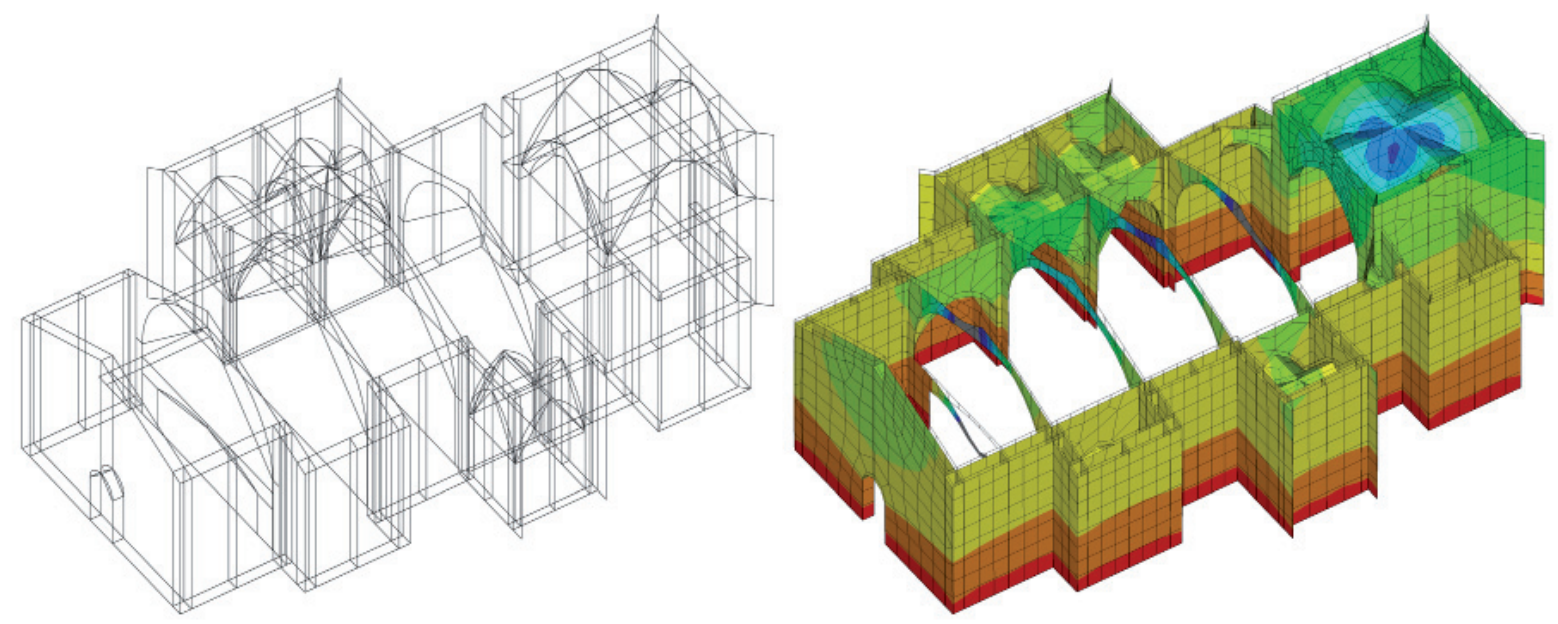

7 Iglesia de santa Mariña, Cambados (Pontevedra, s. XV). Modelo geométrico global y estudio de desplazamientos verticales bajo cargas gravitatorias máximas en situación de servicio. 

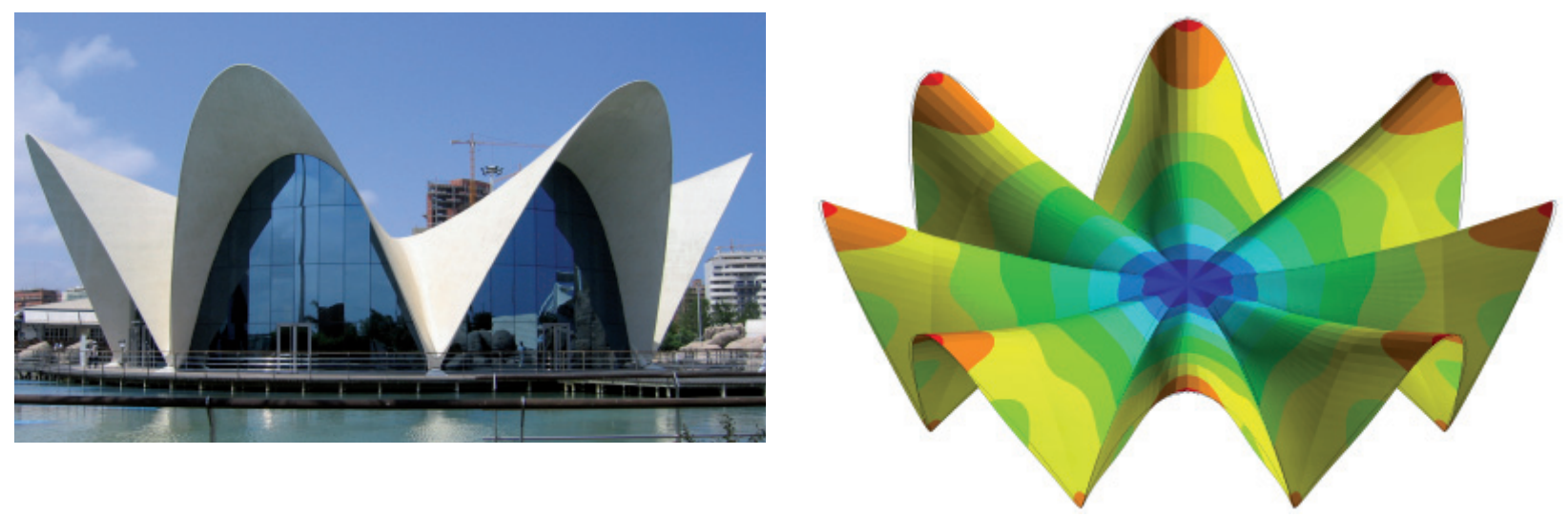

8 Félix Candela Outeiriño, Restaurante Submarino en el Parque Oceanográfico Universal (Valencia, 1999). Modelo de la cubierta laminar definida por la inter-sección de paraboloides hiperbólicos.

zado base sobre el que se desarrolla el modelo. En un primer caso se gestionan polilíneas tridimensionales con un máximo de seis vértices, señalizados de forma consecutiva (Fig. 07). Cabe señalar que si alguna de las aristas que delimitan la superficie debe ser curva, basta trazar previamente el arco que corresponda entre los puntos extremos: el preprocesador detecta automáticamente dicho contorno y ajusta en consecuencia el trazado del área. La alternativa en la confección de áreas viene de los comandos de construcción de caras con cuatro lados y cualquier orientación espacial (mallas poligonales, superficies regladas o de revolución, trazados interpolados entre lados adyacentes, etc.) o del catálogo de entidades tridimensionales básicas del propio entorno $C A D$ (cúpula, esfera, toroide, etc.). En todos estos casos resulta fundamental controlar las variables que definen la densidad de las mallas resultantes en cada dirección, y efectuar una descomposición final que origine $3 \mathrm{~d}$-caras independientes (Fig. 08).

Por último, se hace necesario definir volúmenes elementales con objeto de modelizar elementos de carácter masivo (Fig. 09). En un primer momento utilizamos nuevamente polilíneas, si bien con un total de ocho vértices definidos secuencialmente de acuerdo con el criterio descrito por Ansys. No obstante, la estructura alámbrica resultante de esta técnica dificultaba extraordinariamente la interpretación global del modelo, al no completar todas las aristas necesarias para definir visualmente el contorno del prisma deseado. Para solventar esta cuestión, se ha creado una aplicación adicional en lenguaje Lisp, que permite la confección de volúmenes mediante mallas multiface- ta. De esta forma se genera - con el mismo número de pulsaciones - una imagen completa del prisma, pero que mantiene en cuanto archivo de intercambio una estructura idéntica a una polilínea tridimensional cualquiera.

\section{PARÁMETROS ASOCIADOS Y AJUSTE ULTERIOR DEL MODELO}

Además de la propia definición geométrica del modelo, desde la aplicación $C A D$ es posible manipular determinados parámetros, a efectos de gestionar a través de los mismos ciertas cualidades del modelo mecánico. Ya se ha citado antes la denominación de la capa como una herramienta para asignar tipos de elementos (finitos) y materiales a las entidades que contiene. De esta forma, el programa de conversión detecta automáticamente el total de capas con designación numérica, y permite definir para cada una de ellas las dimensiones resistentes y otros valores relevantes en el análisis estructural, como pueden ser la densidad o el módulo de elasticidad longitudinal. Al respecto del último parámetro, y en el caso de modelizar una construcción de fábrica, es posible utilizar el módulo equivalente que, de acuerdo con la bibliografía ${ }^{6}$, se determina en función de las relaciones entre los espesores de tendel e hilada, del cociente de rigideces de ladrillos y morteros, y del coeficiente de fluencia del conjunto.

Se han utilizado propiedades gráficas como el factor de escala del tipo de línea o el grosor de la misma, para asignar posibles acciones distribuidas sobre una determinada alineación, o bien definir el número de 


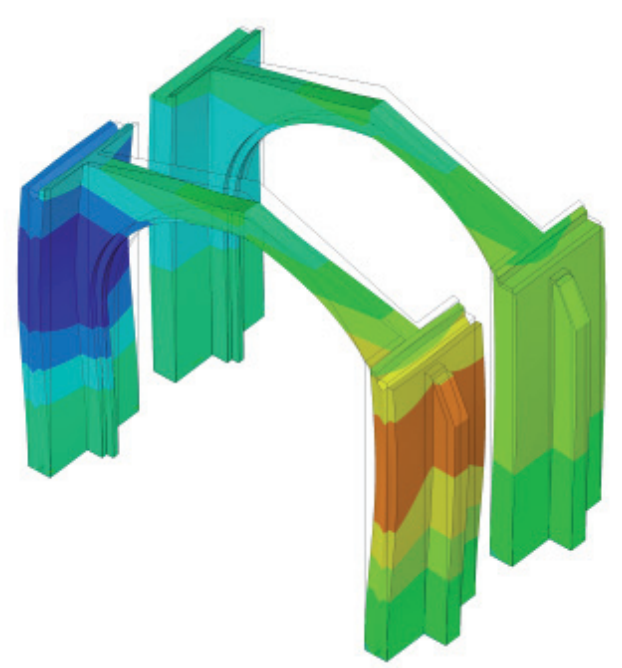

9 Modelo tridimensional que ilustra las deformaciones originadas en un arco fajón por falta de traba con hoja exterior y contrafuerte, unida a la progresiva de-gradación de los rellenos internos del muro compuesto.

divisiones que debe respetarse durante su mallado. Asimismo, se ha considerado la posibilidad de insertar bloques con atributos específicos para aplicar cargas concentradas o vínculos en determinados puntos clave del modelo.

Las especificaciones restantes de la idealización se definen con ayuda del programa de conversión e intercambio, o bien editando directamente el archivo resultante del mismo con ayuda de cualquier editor de textos convencional. Esta última cuestión constituye una de las fortalezas del método, toda vez que es posible ajustar y calibrar paulatinamente el modelo mediante pequeñas operaciones sobre dicho fichero, sin volver en cada caso a la edición del archivo gráfico original.

\section{EJEMPLO DE APLICACIÓN}

A efectos de ilustrar la utilidad práctica de la metodología descrita, además de los casos ya citados, cabe aportar una breve descripción sobre el modelo de análisis confeccionado para el ascensor panorámico recientemente construido en la localidad de Ribadeo (Lugo) ${ }^{7}$. Como se aprecia en la imagen (Fig. 10), la construcción vincula la zona del puerto deportivo con la ciudad alta, evitando el extenso rodeo a que obligaban los accesos preexistentes. La obra surge de un concurso promovido en 2005 dentro del programa europeo Interreg IIIC, cuyo objetivo era promover soluciones de accesibilidad encuadradas en cascos históricos del sur de Europa.

El proyecto alberga un núcleo de escaleras y un as- censor con paradas inferior, intermedia y superior, salvando un desnivel de aproximadamente veintidós metros. Desde un punto de vista estructural, se conforma mediante un doble tubo de hormigón armado, formando una L invertida. Sus dos brazos presentan múltiples aberturas de diferente tamaño y con una ordenación sensiblemente irregular, que enmarcan vistas panorámicas de indudable atractivo. Adicionalmente, el tubo horizontal se proyecta en vuelo, es decir, evitando su apoyo directo sobre la cima de la ladera con el objeto de no comprometer su estabilidad. Toda esta configuración deriva en un comportamiento estructural de cierta complejidad, cuyo análisis requiere el uso de recursos numéricos avanzados.

El modelo se confecciona en $C A D$, siguiendo el procedimiento descrito en los párrafos precedentes, y se fracciona utilizando elementos finitos de tipo lámina, con ocho nodos sujetos a seis posibles grados de libertad. Como excepción, en aquellas zonas donde dos huecos se proyectaron tangentes por una esquina, se usan barras ficticias de conexión, cuyo efecto se materializaría constructivamente mediante crucetas de acero ocultas.

El análisis muestra estados tensionales que, en conjunto, se encuentran dentro de límites razonables, pero también permite identificar aquellas áreas potencialmente conflictivas: en este sentido, destaca la conexión entre la viga-pared que cierra el vuelo por su lateral derecho y el muro en el que apoya, y que en último término exigió una construcción especialmente cuidadosa. Otros aspectos que fue posible estudiar 


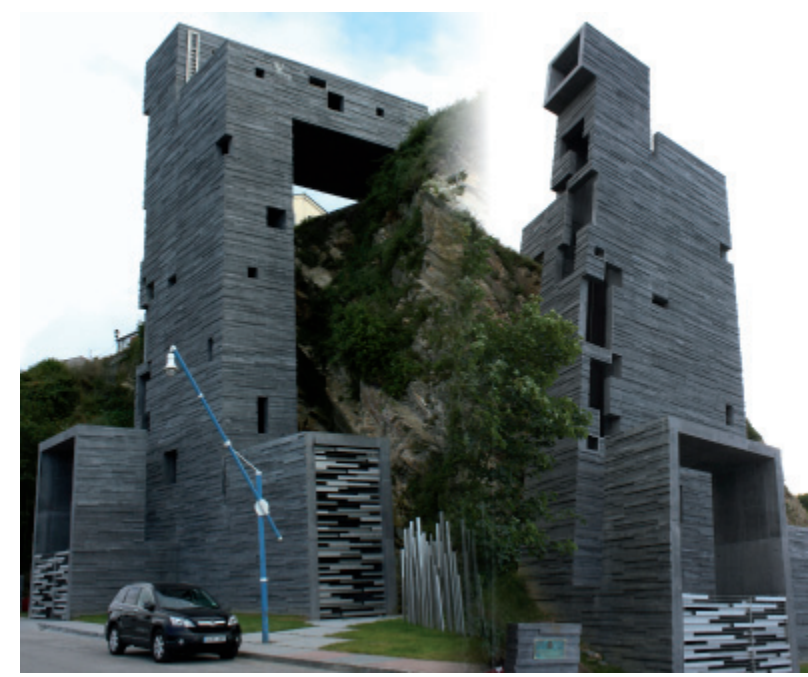

10 Elizabeth Abalo Díaz y Gonzalo Alonso Núñez, Ascensor panorámico, Ribadeo (Lugo, 2010). Fotografía de la obra terminada (arriba) y modelo analítico tridimensional (abajo).

en detalle fueron los estados límite de servicio, con el objeto de mantener las deformaciones por debajo de los límites normativos y de controlar asimismo las posibles vibraciones ocasionadas por el funcionamiento del ascensor y la afluencia de público.

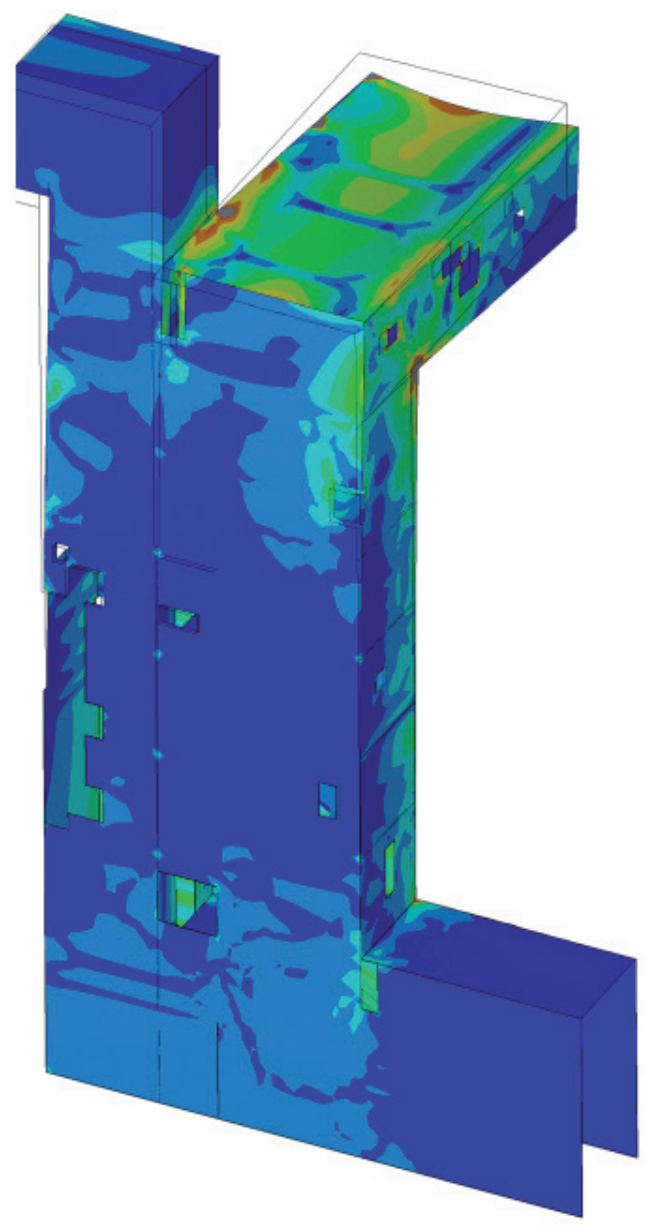

\section{CONCLUSIONES}

El desarrollo de modelos de análisis numérico de cierta complejidad geométrica puede potenciarse extraordinariamente combinando la utilización de aplicaciones específicas de análisis y técnicas de diseño asistido por ordenador. Esta consideración resulta especialmente relevante cuando cabe abordar la confección del modelo sobre levantamientos gráficos previos, como es habitual en el ámbito de la arquitectura.

Para ello, es preciso establecer un mecanismo de conexión entre programas de diferente propósito. De entre las alternativas posibles, se valoran positivamente aquéllas de carácter abierto, fácilmente legibles y manipulables para un usuario no especializado. En este sentido, los archivos de intercambio tipo $D X F$, en su variante $A S C I I$, conllevan una notable simplicidad en su concepción y permiten su tratamiento con herramientas básicas. En nuestra investigación, las hemos utilizado conjuntamente con nuevas rutinas de conversión, para desarrollar archivos susceptibles de ser procesados automáticamente bajo un entorno MEF. La técnica se ha utilizado satisfactoriamente para abordar estudios de estabilidad estructural y valorar posibles procedimientos de intervención, así como para afrontar el análisis de construcciones contemporáneas de cierta singularidad. No obstante, con variaciones coyunturales, puede ser adaptada a otros objetivos o extendida a la conexión entre aplicaciones diferentes de las mencionadas. 


\section{Notas}

1. Cf. Autodesk In., «Documentación de desarrollador: Manual de referencia de DXF», http://exchange.autodesk.com/autocad/esp/help. Consultado el 30 de septiembre de 2011.

2. Cf. Ansys Inc., «Ansys Multiphysics», www.ansys.com/Products/Simulation+Technology/Multiphysics. Consultado el 30 de septiembre de 2011.

3. Cf. Emilio Martín Gutiérrez y Juan B. Pérez Valcárcel, «DxfAnsys v 12.2.3. Generación de modelos de Ansys mediante archivos DXF» (A Coruña: Edición de los autores, 2000). Es posible obtener la última versión del programa (14.02.00) para su libre uso y distribución, a través del correo electrónico de sus autores.

4. Véase, por ejemplo, Juan B. Pérez Valcárcel, «La eficacia estructural de antiguos refuerzos sobre edificios históricos: las iglesias de Guimarei y Ribas de Miño en Lugo, España» (comunicación presentada en el IV Congreso Internacional de Rehabilitación del Patrimonio Arquitectónico y Edificación, La Habana, Cuba, 13-17 de julio de 1998); Idem., «Structural modelling of medieval walls» (comunicación presentada al $3^{\text {rd }}$ International Seminar on Historical Constructions, Possibilities of numerical and experimental techniques, University of Minho, Guimaraes, Portugal, 7-9 de noviembre de 2001); Idem., «Structural behaviour of gothic vaults» (comunicación presentada a la 8th International Conference on Structural Studies, Repairs and Maintenance of Heritage Architecture, Haldikiki, Grecia, 6-7 de mayo de 2003).

5. Cf. Javier Estévez Cimadevila y Emilio Martín Gutiérrez, «Museo del Papel en Carballino. Una estructura de edificación singular», Hormigón y Acero 235 (2005): 35-42.

6. Cf. Code UIC 778-3, Recomendations pour l'evaluation de la capacité portante des ponts-voûtes existants en maçonnerie et beton (Union Internationale des Chemins de Fer, 1995). Véase también José Luis Martínez Martínez, José Antonio Martín-Caro Álamo y Francisco Javier León González, Comportamiento mecánico de la obra de fábrica (Madrid: Universidad Politécnica de Madrid, 2001).

7. Cf. Elizabeth Abalo Díaz y Gonzalo Alonso Núńez, «Conexión y accesibilidad entre el casco histórico de Ribadeo y el borde litoral», en Sebastián Valverde Comesaña y María Balsa Canel (ed.), Soluciones de accesibilidad y movilidad en los centros históricos del sur de Europa (A Coruña: euroGráficas Pichel, 2007), 209-227.

Procedencia de las ilustraciones

Todas las ilustraciones son obra de los autores.

\section{Sobre los autores}

Emilio Martín Gutiérrez. Arquitecto por la Universidad de Santiago de Compostela (1988), con las especialidades de Edificación y Urbanismo. Doctor Arquitecto por la Universidad de A Coruńa (2001), Premio Extraordinario de Doctorado, y Profesor Titular de la misma desde 1990, donde imparte docencia dentro del Departamento de Tecnología de la Construcción. Sus líneas de investigación fundamentales son el desarrollo de soluciones desplegables para el proyecto de cubiertas ligeras, las técnicas de análisis numérico de edificaciones históricas, y la optimización del anclaje de barras metálicas encoladas en piezas de madera. Los resultados de su trabajo han sido publicados en diversos libros, revistas y actas de congresos internacionales. Es autor de varias aplicaciones informáticas de análisis estructural y dispone de una amplia experiencia como consultor de estructuras en el ámbito de la edificación.

emartin@udc.es

Juan B. Pérez Valcárcel. Doctor arquitecto y licenciado en Ciencias Físicas. Catedrático de Estructuras de la Universidad de La Coruña desde 1989. Imparte docencia en la E.T.S. de Arquitectura desde 1977. Es especialista en cálculo de estructuras, siendo pionero en el uso de ordenadores, desde 1974, y habiendo desarrollado numerosos programas de análisis para los tipos estructurales más diversos. En el equipo de investigación que dirige se han desarrollado los primeros métodos para el diseńo y cálculo de estructuras desplegables del mundo, que han sido presentados en diversos foros internacionales con un amplio reconocimiento. También ha desarrollado investigaciones sobre estabilidad estructural en edificios monumentales. Destacan sus estudios sobre la modelización de muros medievales y su influencia en la estabilidad estructural del edificio completo, igualmente presentados en diversos foros internacionales. Ha dirigido ocho tesis doctorales en estas dos líneas de investigación. Es experto en patología y refuerzo de estructuras.

valcarce@udc.es 